\title{
Zero-cycles on varieties over finite fields
}

\author{
Reza Akhtar
}

December 31, 2001

\section{Introduction}

For any field $k$, Milnor [Mi] defined a sequence of groups $K_{0}^{M}(k), K_{1}^{M}(k), K_{2}^{M}(k), \ldots$ which later came to be known as Milnor K-groups. These were studied extensively by Bass and Tate [BT], Suslin [Su], Kato [Ka1], [Ka2] and others. In [Som], Somekawa investigates a generalization of this definition proposed by Kato: given semi-abelian varieties $G_{1}, \ldots, G_{s}$ over a field $k$, there is a group $K\left(k ; G_{1}, \ldots, G_{s}\right)$ which is isomorphic to $K_{s}^{M}(k)$ in the case that $G_{1}=\ldots=G_{s}=\mathbf{G}_{m}$, the multiplicative group scheme. Raskind and Spiess [RS] use a similar idea to define a Milnor-type group $K\left(k ; \mathcal{C H}_{0}\left(X_{1}\right), \ldots, \mathcal{C H}_{0}\left(X_{r}\right)\right)$ associated to a family $X_{1}, \ldots, X_{r}$ of smooth projective varieties over $k$ and prove that this group is isomorphic to the Chow group $C H_{0}\left(X_{1} \times_{k} \ldots \times_{k} X_{r}\right)$ of zero-cycles. These two definitions were amalgamated in [A2] to define groups $K\left(k ; \mathcal{C H}_{0}\left(X_{1}\right), \ldots, \mathcal{C H}_{0}\left(X_{r}\right) ; G_{1}, \ldots, G_{s}\right)$ where the $G_{i}$ are as above and this time $X_{1}, \ldots, X_{r}$ are only assumed to be quasiprojective. In the case $G_{1}=\ldots=G_{s}=\mathbf{G}_{m}$, we use the abbreviated notation $K_{s}\left(k ; \mathcal{C H}_{0}\left(X_{1}\right), \ldots, \mathcal{C H}_{0}\left(X_{r}\right) ; \mathbf{G}_{m}\right)$ for this group.

The main result of $[\mathrm{A} 2]$ is the following:

Theorem 1.1. Let $X$ be a smooth quasiprojective variety of dimension d over a field $k$. Then there are natural isomorphisms:

$$
K_{s}\left(k ; \mathcal{C H}_{0}(X) ; \mathbf{G}_{m}\right) \cong C H^{d+s}(X, s) \cong H_{Z a r}^{d}\left(X, \mathcal{K}_{d+s}^{M}\right)
$$

The middle group above is Bloch's higher Chow group and the (Zariski) sheaf on the far right is the sheaf associated to the presheaf $U \mapsto K_{s}^{M}\left(\mathcal{O}_{X}(U)\right)$.

Using Steinberg's result that the Milnor $K$-groups of finite fields [Mi] vanish in degree $\geq 2$ together with an explicit description of the above cohomology group, we were able to prove the following theorem: 
Theorem 1.2. Let $X$ be a smooth quasiprojective variety of dimension d over a finite field $k$, and suppose $s \geq 2$. Then $C H^{d+s}(X, s)=0$.

The techniques of [A2] do not suffice to investigate the group $C H^{d+1}(X, 1)$, which is the objective of this report. Our main result is:

Theorem 1.3. Let $X$ be a smooth projective variety of dimension d over a finite field with structure morphism $\sigma: X \longrightarrow$ Spec $k$. Then the induced map

$$
\sigma_{*}: C H^{d+1}(X, 1) \longrightarrow C H^{1}(\operatorname{Spec} k, 1)
$$

on higher Chow groups induces an isomorphism of $C H^{d+1}(X, 1)$ with $C H^{1}(\operatorname{Spec} k, 1) \cong k^{*}$.

The results of this paper formed part of my Ph.D. thesis at Brown University. I would like to thank Niranjan Ramachandran in particular for a conversation which sparked the idea for this paper, and also my advisor, Stephen Lichtenbaum, for his constant encouragement and guidance. I also acknowledge the Natural Sciences and Engineering Research Council of Canada for their generous financial support during my years at Brown.

\section{Mixed $K$-groups}

\subsection{Definitions}

In the following we define the "mixed $K$-groups" first introduced in [A2].

Let $k$ be a field, and $X$ a smooth quasiprojective variety defined over $k$. We use the notation $C H_{0}(X)$ for the group of zero-cycles on $X$ modulo rational equivalence. If $G$ is a group scheme defined over $k$ and $A$ is a $k$-algebra, we use the notation $G(A)$ for the group of $A$-rational points, i.e. morphisms Spec $A \longrightarrow G$ which commute with the structure maps.

Let $r \geq 0$ and $s \geq 0$ be integers; let $X_{1}, \ldots, X_{r}$ be smooth quasiprojective varieties defined over $k$ and $G_{1}, \ldots, G_{s}$ semi-abelian varieties defined over $k$. Set

$$
T=\bigoplus_{E / k \text { finite }} C H_{0}\left(\left(X_{1}\right)_{E}\right) \otimes \ldots \otimes C H_{0}\left(\left(X_{r}\right)_{E}\right) \otimes G_{1}(E) \otimes \ldots \otimes G_{s}(E)
$$

We use the notation $\left(a_{1} \otimes \ldots \otimes a_{r} \otimes b_{1} \otimes \ldots \otimes b_{s}\right)_{E}$ to refer to a homogeneous element living in the direct summand of $F$ corresponding to the field $E$.

Define $R \subseteq T$ as the subgroup generated by the elements of the following type: 
- M1. For convenience of notation, set $H_{i}(E)=C H_{0}\left(\left(X_{i}\right)_{E}\right)$ for $i=1, \ldots, r$ and $H_{j}(E)=G_{j-r}(E)$ for $j=r+1, \ldots, r+s$.

For every diagram $k \hookrightarrow E_{1} \stackrel{\phi}{\hookrightarrow} E_{2}$ of finite extensions of $k$, all choices $i_{0} \in$ $\{1, \ldots, r+s\}$ and all choices $h_{i_{0}} \in H_{i_{0}}\left(E_{2}\right)$ and $h_{i} \in H_{i}\left(E_{1}\right)$ for $i \neq i_{0}$, the element $R_{1}\left(E_{1} ; E_{2} ; i_{0} ; h_{1}, \ldots, h_{r+s}\right)$ defined to be:

$$
\left(\phi^{*}\left(h_{1}\right) \otimes \ldots h_{i_{0}} \otimes \ldots \otimes \phi^{*}\left(h_{r+s}\right)\right)_{E_{2}}-\left(h_{1} \otimes \ldots \otimes \phi_{*}\left(h_{i_{0}}\right) \otimes \ldots \otimes h_{r+s}\right)_{E_{1}}
$$

Here we have used the notation $\phi^{*}\left(\phi_{*}\right)$ to denote the pullback (pushforward) map for the Chow group structure on $H_{i}$ (if $1 \leq i \leq r$ ) or the group scheme structure on $H_{i}$ (if $s \leq i \leq r+s$ ).

- M2. For every $K$ finitely generated of transcendence degree 1 over $k$, all choices of $h \in K^{*}, f_{i} \in C H_{0}\left(\left(X_{i}\right)_{K}\right)$ for $i=1, \ldots, r$ and $g_{j} \in G_{j}(K)$ for $j=1, \ldots, s$ such that for every place $v$ of $K$ such that $v(k)=0$, there exists $j_{0}(v)$ such that $g_{j} \in G_{j}\left(O_{v}\right)$ for all $j \neq j_{0}(v)$, the element $R_{2}\left(K, h, f_{1}, \ldots, f_{r}, g_{1}, \ldots, g_{s}\right)$. If $s>0$, this is defined to be:

$$
\sum_{v}\left(s_{v}\left(f_{1}\right) \otimes \ldots \otimes s_{v}\left(f_{r}\right) \otimes g_{1}(v) \otimes \ldots \otimes \tilde{T}_{v}\left(g_{j_{0}(v)}, h\right) \otimes \ldots \otimes g_{s}(v)\right)_{k(v)}
$$

Here $O_{v}$ is the valuation ring of $v, s_{v}: C H_{0}\left(\left(X_{i}\right)_{K}\right) \longrightarrow C H_{0}\left(\left(X_{i}\right)_{k(v)}\right)$ is the specialization map for Chow groups (cf. [F], 20.3), and $g_{i}(v) \in G_{i}(k(v))$ denotes the reduction of $g_{i} \in G_{i}\left(O_{v}\right)$. The notation $\tilde{T}_{v}$ refers to the "extended tame symbol" as defined in [Som]. In the case $G_{1}=\ldots=G_{s}=\mathbf{G}_{m}$, which is our only concern in this paper, this coincides with the (ordinary) tame $v$-adic symbol $T_{v}$. If $s=0$, the element $R_{2}\left(K ; h ; f_{1}, \ldots, f_{r} ; g_{1}, \ldots, g_{s}\right)$ is defined to be:

$$
\sum_{v} \operatorname{ord}_{v}(h)\left(s_{v}\left(f_{1}\right) \otimes \ldots s_{v}\left(f_{r}\right)\right)_{k(v)}
$$

Finally, when $r+s>0$, we define the mixed $K$-group, $K\left(k ; \mathcal{C H}_{0}\left(X_{1}\right), \ldots, \mathcal{C H}_{0}\left(X_{r}\right) ; G_{1}, \ldots, G_{s}\right)$ as the quotient $T / R$. We denote the class of a generator $\left(h_{1} \otimes \ldots \otimes h_{r+s}\right)_{E}$ by $\left\{h_{1}, \ldots, h_{r+s}\right\}_{E / k}$. We refer to the classes of elements of the form M1 and M2 as relations of the mixed $K$-group.

We complete the picture by defining our group to be $\mathbf{Z}$ in the case $r=s=0$. 
We will mostly be interested in the case $G_{1}=\ldots=G_{s}=\mathbf{G}_{m}$; hence we use $K_{s}\left(k ; \mathcal{C H}_{0}\left(X_{0}\right), \ldots, \mathcal{C H}_{0}\left(X_{r}\right) ; \mathbf{G}_{m}\right)$ as shorthand for

$K_{s}(k ; \mathcal{C H}_{0}\left(X_{0}\right), \ldots, \mathcal{C H}_{0}\left(X_{r}\right) ; \underbrace{\mathbf{G}_{m}, \ldots, \mathbf{G}_{m}}_{s})$. We also adopt the practice of omitting superfluous semicolons; for example, if $r=0$, we simply write $K\left(k ; G_{1}, \ldots, G_{s}\right)$ for the group above.

\section{Remark.}

If $\sigma: Y \longrightarrow$ Spec $k$ is a projective variety, we can define the degree map deg $=$ $\sigma_{*}: C H_{0}(Y) \longrightarrow C H_{0}($ Spec $k) \cong \mathbf{Z}$ by push-forward of cycles. We define $A_{0}(Y):=$ Ker deg, and note that if $Y$ contains a $k$-rational point, or more generally if $Y$ admits a zero-cycle of degree 1, then the degree map splits and we have a direct sum decomposition $C H_{0}(Y) \cong \mathbf{Z} \oplus A_{0}(Y)$.

Returning to our situation, suppose that $X_{1}, \ldots, X_{q}$ and $Y_{1}, \ldots, Y_{r}$ smooth quasiprojective, with $Y_{1}, \ldots, Y_{r}$ actually projective. By replacing $C H_{0}$ with $A_{0}$ in the appropriate instances, we may define groups $K\left(k ; \mathcal{C H}_{0}\left(X_{1}\right), \ldots, \mathcal{C H}_{0}\left(X_{q}\right) ; \mathcal{A}_{0}\left(Y_{1}\right), \ldots, \mathcal{A}_{0}\left(Y_{r}\right) ; G_{1}, \ldots, G_{s}\right)$ as was done previously. The aim of the next two sections is to investigate the relationships among these groups.

\section{$2.2 \quad$ Functoriality}

In this section, we list the various functorial properties enjoyed by the mixed $K$-groups introduced above. These properties are exactly analogous to those of [Som], 1.3, so we omit the proofs here and instead refer the interested reader to [A1] for details. The first two properties reflect functoriality in the field, the third functoriality in the semi-abelian varieties, and the latter two functoriality in the quasiprojective varieties. As before, $k$ is a (base) field; $X_{1}, \ldots X_{q}$ are smooth quasiprojective varieties defined over $k ; Y_{1}, \ldots, Y_{r}$ are smooth projective varieties defined over $k$, and $G_{1}, \ldots, G_{s}$ are semiabelian varieties defined over $k$.

Proposition 2.1. (Covariant functoriality in the field) Let $i: k \hookrightarrow k^{\prime}$ be any field extension. Then there exists a natural homomorphism:

$$
\begin{gathered}
i_{*}: K\left(k ; \mathcal{C H}_{0}\left(X_{1}\right), \ldots, \mathcal{C H}_{0}\left(X_{q}\right) ; \mathcal{A}_{0}\left(Y_{1}\right), \ldots, \mathcal{A}_{0}\left(Y_{r}\right) ; G_{1}, \ldots, G_{s}\right) \longrightarrow \\
K\left(k^{\prime} ; \mathcal{C H}_{0}\left(\left(X_{1}\right)_{k^{\prime}}\right), \ldots, \mathcal{C H}_{0}\left(\left(X_{q}\right)_{k^{\prime}}\right) ; \mathcal{A}_{0}\left(\left(Y_{1}\right)_{k^{\prime}}\right), \ldots, \mathcal{A}_{0}\left(\left(Y_{r}\right)_{k^{\prime}}\right) ;\left(G_{1}\right)_{k^{\prime}}, \ldots,\left(G_{s}\right)_{k^{\prime}}\right)
\end{gathered}
$$


Proposition 2.2. (Contravariant functoriality in the field) Let $i: F \hookrightarrow k$ be a finite field extension. Then there exists a natural homomorphism:

$$
\begin{aligned}
& i^{*}: K\left(k ; \mathcal{C H}_{0}\left(X_{1}\right), \ldots, \mathcal{C H}_{0}\left(X_{q}\right) ; \mathcal{A}_{0}\left(Y_{1}\right), \ldots, \mathcal{A}_{0}\left(Y_{r}\right) ; G_{1}, \ldots, G_{s}\right) \longrightarrow \\
& K\left(F ; \mathcal{C H}_{0}\left({ }_{F} X_{1}\right), \ldots, \mathcal{C H}_{0}\left({ }_{F} X_{q}\right) ; \mathcal{A}_{0}\left({ }_{F} Y_{1}\right), \ldots, \mathcal{A}_{0}\left({ }_{F} Y_{r}\right) ;{ }_{F} G_{1}, \ldots,{ }_{F} G_{s}\right)
\end{aligned}
$$

(In the above, ${ }_{F} X_{1}$ denotes $X_{1}$ considered as a scheme over $F$, etc.)

Proposition 2.3. (Morphisms of semi-abelian varieties) Let $G_{j}^{\prime}, j=1, \ldots, s$ be semi-abelian varieties defined over $k$ and $\psi_{j}: G_{j} \longrightarrow G_{j}^{\prime}$ morphisms of semi-abelian varieties over $k$. Then there exists a natural homomorphism:

$$
\begin{gathered}
\psi_{*}: K\left(k ; \mathcal{C H}_{0}\left(X_{1}\right), \ldots, \mathcal{C H}_{0}\left(X_{q}\right) ; \mathcal{A}_{0}\left(Y_{1}\right), \ldots, \mathcal{A}_{0}\left(Y_{r}\right) ; G_{1}, \ldots, G_{s}\right) \longrightarrow \\
K\left(k ; \mathcal{C H}_{0}\left(X_{1}\right), \ldots, \mathcal{C H}_{0}\left(X_{q}\right) ; \mathcal{A}_{0}\left(Y_{1}\right), \ldots, \mathcal{A}_{0}\left(Y_{r}\right) ; G_{1}^{\prime}, \ldots, G_{s}^{\prime}\right)
\end{gathered}
$$

Proposition 2.4. (Covariant functoriality in varieties) Suppose $X_{i}^{\prime} i=1, \ldots, q$ are smooth quasiprojective varieties defined over $k$ and $Y_{j}^{\prime}, j=1, \ldots, r$ smooth projective varieties defined over $k$; suppose furthermore that there are proper morphisms $\phi_{i}$ : $X_{i} \longrightarrow X_{i}^{\prime}$ and $\psi_{j}: Y_{j} \longrightarrow Y_{j}^{\prime}$ for all $i=1, \ldots q$ and $j=1, \ldots, r$. Then pushforward of cycles induces a natural homomorphism:

$$
\begin{gathered}
(\phi, \psi)_{*}: K\left(k ; \mathcal{C H}_{0}\left(X_{1}\right), \ldots, \mathcal{C H}_{0}\left(X_{q}\right) ; \mathcal{A}_{0}\left(Y_{1}\right), \ldots, \mathcal{A}_{0}\left(Y_{r}\right) ; G_{1}, \ldots, G_{s}\right) \longrightarrow \\
K\left(k ; \mathcal{C H}_{0}\left(X_{1}^{\prime}\right), \ldots, \mathcal{C H}_{0}\left(X_{q}^{\prime}\right) ; \mathcal{A}_{0}\left(Y_{1}^{\prime}\right), \ldots, \mathcal{A}_{0}\left(Y_{r}^{\prime}\right) ; G_{1}, \ldots, G_{s}\right)
\end{gathered}
$$

Proposition 2.5. (Contravariant functoriality in varieties) Suppose $X_{i}^{\prime} i=1, \ldots, q$ are smooth quasiprojective varieties defined over $k$ and $Y_{j}^{\prime}, j=1, \ldots, r$ smooth projective varieties defined over $k$; suppose furthermore that there are flat morphisms $\phi_{i}: X_{i} \longrightarrow X_{i}^{\prime}$ and $\psi_{j}: Y_{j} \longrightarrow Y_{j}^{\prime}$ of relative dimension 0 for all $i=1, \ldots q$ and $j=1, \ldots, r$. Then pullback of cycles induces a natural homomorphism:

$$
\begin{gathered}
(\phi, \psi)^{*}: K\left(k ; \mathcal{C H}_{0}\left(X_{1}^{\prime}\right), \ldots, \mathcal{C H}_{0}\left(X_{q}^{\prime}\right) ; \mathcal{A}_{0}\left(Y_{1}^{\prime}\right), \ldots, \mathcal{A}_{0}\left(Y_{r}^{\prime}\right) ; G_{1}, \ldots, G_{s}\right) \longrightarrow \\
K\left(k ; \mathcal{C H}_{0}\left(X_{1}\right), \ldots, \mathcal{C H}_{0}\left(X_{q}\right) ; \mathcal{A}_{0}\left(Y_{1}\right), \ldots, \mathcal{A}_{0}\left(Y_{r}\right) ; G_{1}, \ldots, G_{s}\right)
\end{gathered}
$$




\subsection{Splitting properties}

The key result of this section could be viewed as a generalization of the principle that for any proper variety $Y$ over a field $k$, the exact sequence

$$
0 \longrightarrow A_{0}(Y) \longrightarrow C H_{0}(Y) \stackrel{\text { deg }}{\longrightarrow} \mathbf{Z} \longrightarrow 0
$$

splits if and only if $Y$ admits a zero-cycle of degree one. Once again, we omit the proofs of these facts, all of which are routine, and refer the reader to [A1], Section 5.4. We continue to use the notation of the previous section.

We begin with an elementary but useful lemma which says essentially that the argument $\mathcal{C H}_{0}(k)$ in a mixed $K$-group acts somewhat like an "identity" in that adding or it removing it yields a $K$-group isomorphic to the original.

Lemma 2.6. There is a natural map inducing an isomorphism:

$$
\begin{gathered}
K\left(k ; \mathcal{C H}_{0}(k), \mathcal{C H}_{0}\left(X_{1}\right), \ldots, \mathcal{C H}_{0}\left(X_{q}\right) ; \mathcal{A}_{0}\left(Y_{1}\right), \ldots, \mathcal{A}_{0}\left(Y_{r}\right) ; G_{1}, \ldots, G_{s}\right) \stackrel{\cong}{\longrightarrow} \\
K\left(k ; \mathcal{C H}_{0}\left(X_{1}\right), \ldots, \mathcal{C H}_{0}\left(X_{q}\right) ; \mathcal{A}_{0}\left(Y_{1}\right), \ldots, \mathcal{A}_{0}\left(Y_{r}\right) ; G_{1}, \ldots, G_{s}\right)
\end{gathered}
$$

Our next step is to construct homomorphisms between groups with $\mathrm{CH}_{0}$-type and $A_{0}$-type arguments.

Proposition 2.7. Suppose $1 \leq j_{0} \leq r$, and that $Z$ is a smooth projective variety defined over $k$ admitting a zero-cycle $z$ of degree 1 . For convenience of notation, set

$$
K_{C H}=K\left(k ; \mathcal{C H}_{0}\left(X_{1}\right), \ldots, \mathcal{C H}_{0}\left(X_{q}\right), \mathcal{C H}_{0}(Z) ; \mathcal{A}_{0}\left(Y_{1}\right), \ldots, \mathcal{A}_{0}\left(Y_{r}\right) ; G_{1}, \ldots, G_{s}\right)
$$

and

$$
K_{A}=K\left(k ; \mathcal{C H}_{0}\left(X_{1}\right), \ldots, \mathcal{C H}_{0}\left(X_{q}\right) ; \mathcal{A}_{0}(Z), \mathcal{A}_{0}\left(Y_{1}\right), \ldots, \mathcal{A}_{0}\left(Y_{r}\right) ; G_{1}, \ldots, G_{s}\right)
$$

Then there are natural homomorphisms $j: K_{A} \longrightarrow K_{C H}$ and $p_{z}: K_{C H} \longrightarrow K_{A}$ defined by the formulae:

$$
\begin{gathered}
j\left(\left\{x_{1}, \ldots, x_{q} ; v, y_{1}, \ldots, y_{r} ; g_{1}, \ldots g_{s}\right\}_{E / k}\right)= \\
\left\{x_{1}, \ldots, x_{q} ; v, y_{1}, \ldots, y_{r} ; g_{1}, \ldots, g_{s}\right\}_{E / k}
\end{gathered}
$$

and 


$$
\begin{gathered}
p_{z}\left(\left\{x_{1}, \ldots, x_{q} ; w, y_{1}, \ldots, y_{r} ; g_{1}, \ldots, g_{s}\right\}_{E / k}\right)= \\
\left\{x_{1}, \ldots, x_{q} ; w-\operatorname{deg}_{E}(w) \phi_{E / k}^{*}(z) ; y_{1}, \ldots, y_{r} ; g_{1}, \ldots, g_{s}\right\}_{E / k}
\end{gathered}
$$

where $\phi_{E / k}: k \hookrightarrow E$ is the inclusion.

The fundamental result we are after is the following:

Proposition 2.8. With notation as in the previous proposition, the map $j$ is a split injection (with splitting $p_{z}$ ) inducing a split exact sequence:

$$
0 \longrightarrow K_{A} \stackrel{j}{\longrightarrow} K_{C H} \longrightarrow K_{\mathbf{Z}} \longrightarrow 0
$$

where

$$
K_{\mathbf{Z}}=K\left(k ; \mathcal{C H}_{0}\left(X_{1}\right), \ldots, \mathcal{C H}_{0}\left(X_{q}\right) ; \mathcal{A}_{0}\left(Y_{1}\right), \ldots, \mathcal{A}_{0}\left(Y_{r}\right) ; G_{1}, \ldots, G_{s}\right)
$$

The next statement follows by induction on the previous proposition:

Corollary 2.9. With $k, X_{i}, Y_{i}$ and $G_{i}$ as before, let $Z_{1}, \ldots, Z_{t}$ be smooth projective varieties defined over $k$, each admitting a zero-cycle of degree 1 . To ease notation, write $K_{i_{1}, \ldots, i_{\nu}}$ for the group

$$
K\left(k ; \mathcal{C H}_{0}\left(X_{1}\right), \ldots, \mathcal{C H}_{0}\left(X_{q}\right) ; \mathcal{A}_{0}\left(Z_{i_{1}}\right), \ldots, \mathcal{A}_{0}\left(Z_{i_{\nu}}\right), \mathcal{A}_{0}\left(Y_{1}\right), \ldots, \mathcal{A}_{0}\left(Y_{r}\right) ; G_{1}, \ldots, G_{s}\right)
$$

Then there is a natural map inducing an isomorphism:

$$
\begin{aligned}
K\left(k ; \mathcal{C H}_{0}\left(X_{1}\right), \ldots, \mathcal{C H}_{0}\left(X_{q}\right), \mathcal{C H}_{0}\left(Z_{1}\right), \ldots, \mathcal{C H}_{0}\left(Z_{t}\right) ; \mathcal{A}_{0}\left(Y_{1}\right), \ldots, \mathcal{A}_{0}\left(Y_{r}\right) ; G_{1}, \ldots, G_{s}\right) \\
\stackrel{\cong}{\bigoplus} \bigoplus_{0 \leq \nu \leq t} \bigoplus_{1 \leq i_{1}<\ldots<i_{\nu} \leq r} K_{i_{1}, \ldots, i_{\nu}}
\end{aligned}
$$

The next result appears as part of the proof of [RS], Theorem 2.4. We recover it directly from the above without explicit mention of motives; all we need is the (functorial) identification of the group of zero-cycles of degree zero on a smooth projective curve with the group of rational points on its Jacobian.

Corollary 2.10. Let $C_{1}, \ldots, C_{t}$ be smooth projective curves over a field $k$, each containing a k-rational point. Let $J_{i}$ denote their respective Jacobians. Then there is an isomorphism:

$$
K\left(k ; \mathcal{C H}_{0}\left(C_{1}\right), \ldots, \mathcal{C H}_{0}\left(C_{t}\right)\right) \cong \bigoplus_{0 \leq \nu \leq t} \bigoplus_{1 \leq i_{1}<\ldots<i_{\nu} \leq t} K\left(k ; J_{i_{1}}, \ldots, J_{i_{t}}\right)
$$




\section{Zero-cycles over finite fields}

In preparation for the statement and proof of the main theorem, we overview some established results.

Theorem 3.1. (Steinberg, [Mi]) Let $k$ be a finite field. For $s \geq 2, K_{s}^{M}(k)=0$.

The analogue for Kato-Somekawa groups is:

Theorem 3.2. (Kahn, [Kahn]) Let $k$ be a finite field and $G_{1}, \ldots, G_{s}$ semi-abelian varieties defined over $k$. Then for $s \geq 2, K\left(k ; G_{1}, \ldots, G_{s}\right)=0$.

We also recall the main theorem of [A2]:

Theorem 3.3. Let $k$ be a field, $s \geq 0$ an integer, and $X$ a smooth quasiprojective variety of dimension $d$ defined over $k$. Then there is a natural map inducing an isomorphism:

$$
\alpha=\alpha_{X}: C H^{d+s}(X, s) \stackrel{\cong}{\longrightarrow} K_{s}\left(k ; \mathcal{C H}_{0}(X) ; \mathbf{G}_{m}\right)
$$

An important result of Lang and Weil is of special relevance to our situation.

Theorem 3.4. ([Sou], 1.5.3 Lemme 1) Let $\sigma: X \longrightarrow$ Spec $k$ be a smooth projective variety where $k$ is a finite field. Then $X$ admits a zero-cycle of degree 1.

With notation as in Theorem 3.4, let $z$ be a zero-cycle of degree 1 on $X$. Furthermore, let $d=\operatorname{dim} X$ and $s \geq 0$ be a integer. Using the product structure on the higher Chow groups, we may define a map $B_{z}: C H^{s}(k, s) \longrightarrow C H^{d+s}(X, s)$ by $B_{z}(x)=z \times x$. Evidently we have $\sigma_{*} \circ B_{z}=i d$, so $B_{z}$ furnishes a splitting of $\sigma_{*}$.

Having fixed a choice of zero-cycle $z$, we have the following consequence of Theorems 3.3 and 3.4 :

Corollary 3.5. Let notation be as in Theorem 3.4; set $d=\operatorname{dim} X$, and let $s \geq 0$ be an integer. Then all squares in the diagram below commute, the map on kernels being induced by the various maps $\alpha$ :

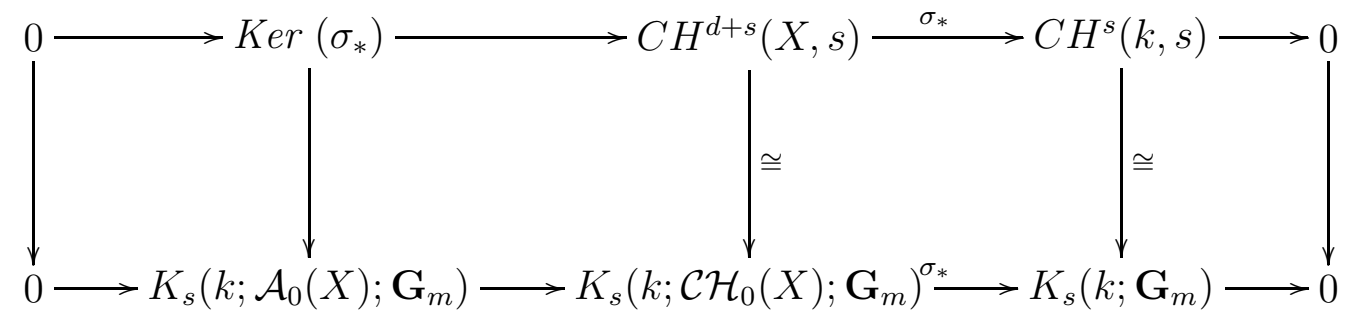

Furthermore, both sequences above are split exact. 
Note that the top sequence is split exact by the preceding discussion, and the bottom sequence by Proposition 2.8. Thus, we have a functorial isomorphism $\operatorname{Ker}\left(\sigma_{*}\right) \cong$ $K_{s}\left(k ; \mathcal{A}_{0}(X) ; \mathbf{G}_{m}\right)$.

Our contribution is the following:

Theorem 3.6. Let $k$ be a finite field and $X$ a smooth projective variety defined over $k$. Then for $s \geq 1, K_{s}\left(k ; \mathcal{A}_{0}(X) ; \mathbf{G}_{m}\right)=0$.

Note that in the case $s \geq 2$, most of the work has already been done. Corollary 3.5 enables us to identify $K_{s}\left(k ; \mathcal{A}_{0}(X) ; \mathbf{G}_{m}\right)$ with a direct summand of the group $K_{s}\left(k ; \mathcal{C H}_{0}(X) ; \mathbf{G}_{m}\right) \cong C H^{d+s}(X, s)$; the latter is zero by Corollary 7.1 of [A2].

It is also worth noting that when $s=1$ and $X$ is a curve containing a rational point, the desired result follows from Theorem 3.2. The group $K\left(k ; \mathcal{A}_{0}(X) ; \mathbf{G}_{m}\right)$ may be identified with the (Kato-Somekawa) group $K\left(k ; J, \mathbf{G}_{m}\right)$, where $J$ is the Jacobian of $X$; then Theorem 3.2 enables us to conclude that this group vanishes.

The general case of Theorem 3.6 will follow from the next Lemma. The idea for the proof is based on that of Kahn [Kahn].

Lemma 3.7. For convenience of notation, let $A=K\left(k ; \mathcal{A}_{0}(X) ; \mathbf{G}_{m}\right)$.

1. A is torsion

2. $A$ is divisible

3. For every prime l, there exists an integer $m_{l}>0$ which annihilates the l-primary torsion subgroup $A\{l\} \subseteq A$.

\section{Proof.}

Throughout this proof we reserve the notation $\phi_{E / k}$ for the map Spec $E \longrightarrow$ Spec $k$ associated to a field extension $E / k$.

Since $A$ is a quotient of $\bigoplus_{E / k}$ finite $A_{0}\left(X_{E}\right) \otimes E^{*}$ and $E^{*}$ is a finite group for each $E / k, A$ must be torsion.

Now let $\{x, y\}_{E / k}$ be a homogeneous element of the group $A$ and suppose $n>0$ is an integer. Since $x \in A_{0}\left(X_{E}\right)$ and $A_{0}(\bar{X})$ is divisible by a result of Bloch ([Bl2], Lemma 1.3), we may choose a finite extension $E^{\prime} / E$ such that $\phi_{E^{\prime} / E}^{*}(x)=n x^{\prime}$ for some $x^{\prime}=A_{0}\left(X_{E^{\prime}}\right)$. Since $E$ is a finite field, it is $C^{1}$ (cf. [Se], 10.7), which implies that the field norm map $F^{*} \longrightarrow E^{*}$ is surjective for any finite extension $F / E$. Thus we may choose $z \in\left(E^{\prime}\right)^{*}$ such that $N_{E^{\prime} / E}(z)=y$. By a relation of type M1, we have

$$
\{x, y\}_{E / k}=\left\{x, N_{E^{\prime} / E}(z)\right\}_{E / k}=\left\{n x^{\prime}, z\right\}_{F / k}=n\left\{x^{\prime}, z\right\}_{F / k}
$$


and so $A$ is divisible.

Towards a proof of the third assertion, suppose $l=p=$ char $k$. Then a typical generator of $A$ is a symbol $\{x, y\}_{E / k}$, where $y=E^{*}$. Since $y$ has order prime to $p$, so does $\{x, y\}_{E / k}$; hence, $A\{p\}=0$.

To treat the case $l \neq$ char $k$, we will need to make use of a number of powerful results, which we collect together in the next theorem. The proof continues afterward, via a sequence of lemmas.

Theorem 3.8. 1. (Kato-Saito, $[K S]$ )

Let $F$ be a finite field and $X$ a smooth projective variety defined over $F$. Then $A_{0}(X)$ is a finite group.

2. (Weil conjectures)

Let $F$ be a finite field and $A$ an abelian variety defined over $F$. Let $\bar{F}$ denote the algebraic closure of $F$ and $\bar{X}$ the extension of $A$ to $\bar{F}$. Suppose $l$ is a prime not equal to char $F$. Then the Frobenius automorphism $\phi \in G a l(\bar{F} / F)$ acts $\mathbf{T}_{l}\left(A_{0}(\bar{X})\right) \otimes_{\mathbf{z}_{l}} \mathbf{T}_{l}\left(\mathbf{G}_{m}\right)$; here $\mathbf{T}_{l}\left(A_{0}(\bar{X})\right)=\stackrel{\lim }{\leftarrow} A_{0}(\bar{X})\left[l^{n}\right]$ and $\mathbf{T}_{l}\left(\mathbf{G}_{m}\right)=\stackrel{\lim }{\leftarrow} \mu_{l^{n}}$, the ordinary Tate module of $\bar{F}$.

3. (Rojtman-Bloch, [Ro], [Bl1])

Let $L$ be an algebraically closed field and $X$ a smooth projective variety defined over $L$. Then the Albanese map $A_{0}(X) \longrightarrow A l b(X)(L)$ induces an isomorphism on torsion subgroups.

Lemma 3.9. The group $A=K\left(k ; \mathcal{A}_{0}(X) ; \mathbf{G}_{m}\right)$ is a quotient of $\bigoplus_{E / k \text { finite }} H_{0}\left(\operatorname{Gal}(E / k), A_{0}\left(X_{E}\right) \otimes E^{*}\right)$.

\section{Proof.}

By construction, $A$ is a quotient of $\bigoplus_{E / k}$ finite $A_{0}\left(X_{E}\right) \otimes E^{*}$. Furthermore, for every $x \in A_{0}\left(X_{E}\right), y \in E^{*}$ and every automorphism $\sigma: E \longrightarrow E$ of $E$ over $k$, the element $x \otimes y=x \otimes \sigma_{*}\left(\sigma^{*}(y)\right)$ is equivalent in $A$ (modulo a relation of type M1) to $\sigma^{*}(x) \otimes \sigma^{*}(y)$. These are exactly the relations by which one defines the zeroth homology group.

Lemma 3.10. Let $G=\operatorname{Gal}(\bar{k} / k)$. Then $H_{0}\left(G, \mathbf{T}_{l}\left(A_{0}(\bar{X})\right) \otimes_{\mathbf{z}_{l}} \mathbf{T}_{l}\left(\mathbf{G}_{m}\right)\right)$ is a finite group.

\section{Proof.}


First, we claim that the Frobenius automorphism $\phi \in G$ acts (diagonally) without fixed points on $\mathbf{T}\left(A_{0}(\bar{X})\right) \otimes \mathbf{z}_{l} \mathbf{T}\left(\mathbf{G}_{m}\right)$. To analyze the action of $\phi$ on the first factor, we need to use Theorem 3.8, part 3, which gives a functorial isomorphism $A_{0}(\bar{X})\left[l^{n}\right] \cong \operatorname{Alb}(\bar{X})(\bar{k})\left[l^{n}\right]$ for all $n$. Taking inverse limits, we have a natural isomorphism $\mathbf{T}\left(A_{0}(\bar{X})\right) \cong \mathbf{T}(\operatorname{Alb}(\bar{X}))$. By the Weil conjectures (Theorem 3.8, part 2), $\phi$ acts without fixed points on the latter module, hence also on the former. The action of $\phi$ on $\mathbf{T}\left(\mathbf{G}_{m}\right) \cong \mathbf{Z}_{l}$ is simply multiplication by $l$, which is an action without fixed points. Hence the diagonal action of $\phi$ on $M=\mathbf{T}_{l}\left(A_{0}(\bar{X})\right) \otimes_{\mathbf{Z}_{l}} \mathbf{T}_{l}\left(\mathbf{G}_{m}\right)$ has no fixed points.

Therefore, $\phi-i d$ is an invertible $\mathbf{Q}_{l}$-linear automorphism of the $\mathbf{Q}_{l}$-vector space $M \otimes \mathbf{z}_{l} \mathbf{Q}_{l}$. Since $H_{0}(G, M) \otimes \mathbf{z}_{l} \mathbf{Q}_{l}$ is a quotient of $\frac{M}{(\phi-i d) M} \otimes \mathbf{z}_{l} \mathbf{Q}_{l}=0$, we conclude that $H_{0}(G, M) \otimes \mathbf{z}_{l} \mathbf{Q}_{l}$ must itself be 0 . Hence $H_{0}(G, M)$ is a torsion $\mathbf{Z}_{l}$-module; furthermore, being a quotient of a finitely generated $\mathbf{Z}_{l}$-module, it is finitely generated. We conclude that $H_{0}(G, M)$ is finite.

Lemma 3.11. For every finite extension $E / k,\left(A_{0}\left(X_{E}\right) \otimes E^{*}\right)\{l\}$ is a quotient of a finite number of copies of $M$.

\section{Proof.}

Clearly $E^{*}$ is a finite group, and $A_{0}\left(X_{E}\right)$ is finite by Theorem 3.8, Part 1. Thus $A_{0}\left(X_{E}\right)\{l\}$ be may written as a finite direct sum $\mathbf{Z} / l^{c_{1}} \mathbf{Z} \oplus \ldots \oplus \mathbf{Z} / l^{c_{m}} \mathbf{Z}$ of cyclic $l$-groups. By choosing $a \geq \max \left(c_{1}, \ldots, c_{m}\right)$ and $n$ sufficiently large, we may thus realize $A_{0}\left(X_{E}\right)\{l\}$ as a subgroup of $\left(\left(\mathbf{Z} / l^{a} \mathbf{Z}\right)^{2 g}\right)^{n} \cong\left(\operatorname{Alb}(\bar{X})\left[l^{a}\right]\right)^{n} \cong\left(A_{0}(\bar{X})\left[l^{a}\right]\right)^{n}$. Using duality, $A_{0}\left(X_{E}\right)\{l\}$ may also be realized as a quotient of $\left(A_{0}(\bar{X})\left[l^{a}\right]\right)^{n}$; since the latter is a quotient of $\mathbf{T}\left(A_{0}(\bar{X})\right)^{n}$, we conclude that $A_{0}\left(X_{E}\right)\{l\}$ is a quotient of some power of $\mathbf{T}_{l}\left(A_{0}(\bar{X})\right)$.

An analogous (simpler) argument shows that $E^{*}\{l\}$ is a quotient of some power of $\mathbf{T}_{l}\left(\mathbf{G}_{m}\right)$. Hence, we conclude that $A_{0}\left(X_{E}\right)\{l\} \otimes_{\mathbf{z}_{l}} E^{*}\{l\} \cong\left(A_{0}\left(X_{E}\right) \otimes_{\mathbf{z}_{l}} E^{*}\right)\{l\}$ is a quotient of some power of $M=\mathbf{T}_{l}\left(A_{0}(\bar{X})\right) \otimes \mathbf{z}_{l} \mathbf{T}_{l}\left(\mathbf{G}_{m}\right)$.

Finally, since $A_{0}\left(X_{E}\right)\{l\}$ and $E^{*}\{l\}$ are finite abelian $l$-groups, their tensor product as $\mathbf{Z}_{l}$-modules coincides (as an abelian group) with their tensor product as $\mathbf{Z}$-modules; therefore we conclude that $\left(A_{0}\left(X_{E}\right) \otimes E^{*}\right)\{l\}$ is a quotient of some power of $M$.

Lemma 3.12. There exists an integer $m_{l}$ which kills $\bigoplus_{E / k}$ finite $H_{0}\left(G_{E / k}, A_{0}\left(X_{E}\right) \otimes\right.$ $\left.E^{*}\right)\{l\}$. 
From Lemma 3.11 and the right exactness of the functor $H_{0}$, we deduce the existence of a surjective map $H_{0}\left(G, M^{n}\right) \longrightarrow H_{0}\left(G,\left(A_{0}\left(X_{E}\right) \otimes E^{*}\right)\{l\}\right)$. By Shapiro's Lemma we may identify the latter group with $H_{0}\left(\mathrm{Gal}(E / k),\left(A_{0}\left(X_{E}\right) \otimes E^{*}\right)\{l\}\right)=$ $H_{0}\left(\operatorname{Gal}(E / k), A_{0}\left(X_{E}\right) \otimes E^{*}\right)\{l\}$.

Set $m_{l}=\# H_{0}(G, M)$. By Lemma 3.10 and the fact that $H_{0}$ commutes with direct sums, $m_{l}$ kills $H_{0}\left(G, M^{n}\right)$, and therefore (by the above argument) $m_{l}$ kills $H_{0}\left(\mathrm{Gal}(E / k), A_{0}\left(X_{E}\right) \otimes E^{*}\right)\{l\}$ for any finite extension $E / k$. We conclude that $m_{l}$ kills the direct sum in the statement of the lemma.

The proof of Lemma 3.7 is finished by the next and last lemma:

Lemma 3.13. $A\{l\}$ is killed by $m_{l}$.

Fix $a \in A\{l\}$. Then $a$ is represented by some element $a^{\prime} \in H_{0}\left(\mathrm{Gal}(E / k), A_{0}\left(X_{E}\right) \otimes\right.$ $\left.E^{*}\right)$ where $E / k$ is some finite extension. If the order of $a^{\text {prime }}$ is prime to $l$, then the order of $a$ (which divides the order of $a^{\prime}$ ) must also be prime to $l$. The assumption that $a \in A\{l\}$ forces $a=0$, which is trivially killed by $m_{l}$. If $l$ divides the order of $a^{\prime}$, then for some $c>1, \operatorname{gcd}(c, l)=1, c a^{\prime} \in H_{0}\left(\operatorname{Gal}(E / k), A_{0}\left(X_{E}\right) \otimes E^{*}\right)\{l\}$; by Lemma 3.12, $m_{l} c a^{\prime}=0$, and hence $m_{l} c a=0$. Finally, the assumption that $a$ is killed by some power $l^{b}$ enables us to conclude that $a$ is killed by $g=\operatorname{gcd}\left(l^{b}, m_{l} c\right)$. Since $g|l, g| m_{l} c$, and $\operatorname{gcd}(c, l)=1$, this forces $g \mid m_{l}$. Thus $a$ is killed by $m_{l}$.

This concludes the proof of the third assertion of Lemma 3.7 and hence also of Theorem 3.6.

Corollary 3.14. Let $X$ be a smooth projective variety of dimension d over a finite field with structure morphism $\sigma: X \longrightarrow$ Spec $k$. Then the map

$$
\sigma_{*}: C H^{d+1}(X, 1) \longrightarrow C H^{1}(\operatorname{Spec} k, 1)
$$

on higher Chow groups induces an isomorphism of $C H^{d+1}(X, 1)$ with $C H^{1}(\operatorname{Spec} k, 1)$ $\cong k^{*}$.

\section{Proof.}

By Corollary 3.5, $\sigma_{*}$ is surjective; its kernel is isomorphic to $K\left(k ; A_{0}(X) ; \mathbf{G}_{m}\right)$, which is zero by Theorem 3.6 .

\section{Some results on one-cycles}

It is natural to ask whether the techniques of the previous section may be extended to study higher-dimensional cycles on varieties over a finite field. This does not seem 
likely: one of the key facts implicit in the above treatment is that (at least in the case of an algebraically closed base field) every element (zero-cycle) of $\mathrm{CH}^{d+s}(X, s)$ is decomposable, i.e. lies in the image of the product map

$$
C H_{0}(X) \otimes\left(k^{*}\right)^{\otimes s}=C H^{d}(X) \otimes C H^{1}(k, 1)^{\otimes s} \longrightarrow C H^{d+s}(X, s)
$$

where the notation is as before. For higher-dimensional cycles, it is simply not true that every cycle is thus decomposable. Nevertheless, it is possible to deduce certain results of a weaker nature. We begin by studying a specific group of one-cycles on curves:

Proposition 4.1. Let $k$ be a finite field and $C$ a smooth curve defined over $k$. Then $C H^{2}(C, 2)$ is finite.

\section{Proof.}

Let $K$ be the function field of $C$. A slightly generalized version of Bloch's localization theorem ([A2], Corollary 4.4) yields an exact sequence

$$
\bigoplus_{z \in C^{1}} C H^{1}(k(z), 2) \longrightarrow C H^{2}(C, 2) \longrightarrow C H^{2}(K, 2) \stackrel{\partial}{\longrightarrow} \bigoplus_{z \in C^{1}} C H^{1}(k(z), 1) \longrightarrow \ldots
$$

Each group in the direct sum on the left vanishes by [Bl3], Lemma 6.3; furthermore, the following diagram commutes, in which the vertical arrows are the isomorphisms of [To], Theorem 1, and the lower horizontal arrow $\partial^{M}$ is the boundary map of Milnor $K$-theory, cf. [A2], 2.2.

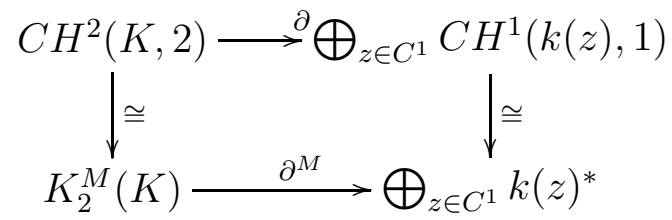

Thus we may identify $C H^{2}(C, 2)$ with $\operatorname{Ker}\left(\partial^{M}: K_{2}^{M}(K) \longrightarrow \bigoplus_{z \in C^{1}} k(z)^{*}\right)$, which is finite by [BT], II. Theorem 2.1.

A weaker, more general result may be deduced using the coniveau spectral sequence for higher Chow groups introduced by Bloch in [Bl3].

Proposition 4.2. Let $k$ be a finite field and $X$ a smooth quasiprojective variety of dimension $d$ over $k$. Then for all $s \geq 2$, the group $C^{d+s-1}(X, s)$ of 1-cycles is a torsion group. 


\section{Proof.}

The coniveau spectral sequence reads:

$$
E_{2}^{p, q}=H_{Z a r}^{p}\left(X, \mathcal{C H}^{d+s-1}(-q)\right) \Longrightarrow C H^{d+s-1}(-p-q)
$$

We are interested in the case $-p-q=s$, or $q=-s-p$. To prove that $C H^{d+s-1}(s)$ is torsion, it is sufficient to show that the terms $E_{\infty}^{p,-s-p}$ are torsion for all $p$. Furthermore, since $E_{n+1}^{p, q}$ is a subquotient of $E_{n}^{p, q}$ for all $p, q$, and $n$, it is sufficient to show that

$$
E_{2}^{p,-s-p}=H_{Z a r}^{p}\left(X, \mathcal{C} \mathcal{H}^{d+s-1}(p+s)\right)
$$

is torsion for all $p$.

If $p>d$, this (cohomology) group is zero for reasons of dimension. If $p<d-1$, the Zariski sheaf $\mathcal{C H}^{d+s-1}(p+s)$ is zero by the Gersten resolution for higher Chow groups ([Bl3], Theorem 10.1). It remains to consider the cases $p=d-1$ and $p=d$. When $p=$ $d-1$, we have $E_{2}^{d-1,-s-d+1}=H_{Z a r}^{d-1}\left(X, \mathcal{C H}^{d+s-1}(d+s-1)\right)$. By the Gersten resolution, this is a subquotient of $\bigoplus_{x \in X^{d-1}} C H^{s}(k(x), s)=\bigoplus_{x \in X^{d-1}} K_{s}^{M}(k(x))$; however, since $s \geq 2$ and $k(x)$ is a global field (of positive characteristic), this latter group (and hence its subquotient, too) is a torsion group. In the case $p=d$, the group to consider is $E_{2}^{d, d+s}=H_{Z a r}^{d}\left(X, \mathcal{C H}_{0}{ }^{d+s-1}(d+s)\right)$. By the Gersten resolution, this is a subquotient of $\bigoplus_{x \in X^{d}} C H^{s-1}(k(x), s)$. In each term $C H^{s-1}(k(x), s)$ is, modulo torsion, isomorphic via the Riemann-Roch Theorem for higher algebraic $K$-theory $([\mathrm{Bl}]])$ to a direct summand of the (algebraic) $K$-group $K_{s}(k(x))$. Since $k(x)$ is a finite field and $s \geq 2$, the latter group is torsion by Quillen's computations of the $K$-theory of finite fields [Qu]. Thus our term $E_{2}^{d, d+s}$ is itself torsion, which concludes the proof.

From the above, we recover the following (much weaker form of) results of Soule [Sou]:

Corollary 4.3. Let $C$ be a smooth projective curve defined over a finite field $k$. Then $K_{1}(C)$ and $K_{2}(C)$ are torsion groups.

\section{Proof.}

Using the Riemann-Roch Theorem, we are reduced to showing that $C H^{i}(C, 1)$ and $C H^{i}(C, 2)$ are torsion for all $i$. For $i=0$, this is trivial in both cases, and when $i=1$, we have $C H^{1}(C, 1) \cong k^{*}$ and $C H^{1}(C, 2)=0$; When $i=2, C H^{2}(C, 1) \cong k^{*}$ by Corollary 3.14 and $C H^{2}(C, 2)$ is finite by Proposition 4.1. For $i=3, C H^{3}(C, 1)=0$ for reasons of dimension and $C H^{3}(C, 2)=0$ by [A2], Corollary 7.1. Finally, in the case $i>3$, both groups vanish for reasons of dimension. 
The case $s=1$ has been excluded from the statement of Proposition 4.2; however, we still expect the group $C H^{d}(X, 1)$ to be torsion when $X$ is projective (and smooth). For curves this is clear, as we have $C H^{1}(X, 1) \cong k^{*}$, which is finite. A conjecture due to Parshin asserts that for $i \geq 1$, the groups $K_{i}(X)$ (and hence also $C H^{j}(X, i)$ ) are torsion for $X$ a smooth projective variety defined over a finite field. The validity of this conjecture is known when $K_{i}$ is a member of a special class of varieties including all products of curves, abelian varieties, unirational varieties and Fermat curves [Sou]. However, even in the case of a general surface $X$, we do not yet know that $C H^{2}(X, 1)$ is torsion. Such a result would be of interest, as it would automatically imply that $C H^{2}(X, 1)$ were finite: by results of Müller-Stach and Elbaz-Vincent ([MSEV], Corollary 5.2) we may identify $C H^{2}(X, 1)$ with $H_{\text {Zar }}^{1}\left(X, \mathcal{K}_{2}\right)$, and the latter is known by a Theorem of Gros and Suwa ([GS], Théorème 4.19) to be an extension of a finite group by a uniquely divisible group. 


\section{References}

[A1] R. Akhtar, Milnor K-theory and zero-cycles on algebraic varieties, Ph.D. Thesis, Brown University, 2000.

[A2] R. Akhtar, Milnor K-theory of smooth quasiprojective varieties, preprint.

[BT] H. Bass and J. Tate, The Milnor ring of a global field, Lecture Notes in Math. 342 (1973), 349-446.

[Bl1] S. Bloch, Torsion algebraic cycles and a theorem of Roitman, Compositio Math. 39 (1979), 107-127.

[Bl2] S. Bloch, Lectures on Algebraic Cycles, Duke University Mathematics Series IV, Duke University, 1980.

[Bl3] S. Bloch, Algebraic cycles and higher K-theory, Adv. Math. 61 (1986), 267304.

[F] W. Fulton, Intersection Theory, Springer-Verlag, 1984.

[GS] M. Gros and N. Suwa, Application d'Abel-Jacobi p-adique et cycles algébriques, Duke Math. J. 55 (1988), no. 2, 579-613.

[Kahn] B. Kahn, Nullité de certains groupes attachés aux variétés semi-abéliennes sur un corps fini; application, C. R. Acad. Sci. Paris Sér. I Math. 314 (1992), no. 13, 1039-1042.

[Ka1] K. Kato, A generalization of local class field theory by using K-group II, J. Fac. Sci. Univ. Tokyo, Sec. IA 27 (1983), 603-683.

[Ka2] K. Kato, Milnor K-theory and the Chow group of zero cycles, Contemp. Math. 55 (1986), 241-253.

[KS] K. Kato and S. Saito, Unramified class field theory of arithmetic surfaces, Ann. Math. 118 (1983), 241-275.

[Mi] J. Milnor, Algebraic K-theory and quadratic forms, Invent. Math. 9 (19691970), 318-344.

[MSEV] S. Müller-Stach and P. Elbaz-Vincent, Milnor K-theory, Higher Chow groups and Applications, preprint. 
[Qu] D. Quillen, Higher Algebraic K-theory I Lecture Notes in Math. 341 (1973), $85-147$.

[RS] W. Raskind and M. Spiess, Milnor K-groups and zero-cycles on products of curves over p-adic fields, Compositio Math. 121 (2000), no. 1, 1-33.

[Ro] A. A. Rojtman, The torsion of the group of 0-cycles modulo rational equivalence, Ann. Math. 111 (1980), no. 3, 553-569.

[Se] J-P. Serre, Corps Locaux, Hermann, 1962.

[Som] M. Somekawa, On Milnor K-groups attached to semiabelian varieties, $K$ theory 4 (1990), 105-119.

[Sou] C. Soulé, Groupes de Chow et K-théorie, Math. Ann. 268, vol. 1, (1984), $317-345$.

[Su] A. A. Suslin, Reciprocity laws and the stable rank of rings of polynomials, Izv. Math. 43 (1979), no. 6, 1394-1429.

[To] B. Totaro, Milnor K-theory is the simplest part of algebraic K-theory, Ktheory 6 (1992), 177-189.

Reza Akhtar

Department of Mathematics and Statistics

Miami University

Oxford, $\mathrm{OH} 45056$

reza@calico.mth.muohio.edu 